\title{
VON HIPPEL-LINDAU DISEASE: THE CLINICAL MANIFESTATIONS AND GENETIC ANALYSIS RESULTS OF TWO CASES FROM A SINGLE FAMILY
}

\author{
Kinyas $\mathrm{S}^{1}$, Ozal SA ${ }^{1, *}$, Guclu $\mathrm{H}^{1}$, Gurlu $\mathrm{V}^{1}$, Esgin $\mathrm{H}^{1}$, Gurkan $\mathrm{H}^{2}$
}

*Corresponding Author: Dr. Sadık Altan Ozal, Trakya Üniversitesi Tıp Fakültesi, Göz Hastalıkları Anabilim Dal1, Edirne 22030, Turkey. Tel: +90-505-450-42-67. Fax: +90-284-223-55-06. E-mail: altanozal@hotmail.com

\begin{abstract}
von Hippel-Lindau (VHL) disease is an autosomal dominant inherited multi systemic cancer syndrome that is classically associated with neoplasms in multiple organs, and caused by mutations in the VHL gene on chromosome 3p25-p26. Retinal hemangioblastoma $(\mathrm{RH})$ is the most frequent and the earliest clinical sign of the disease, which is seen in $40.0-60.0 \%$ of patients. In recent years, studies of patients with VHL tried to put forward the relationship between genotype and phenotype. In this study, two VHL cases in the same family with clinical findings and genetic analysis results are presented. As a consequence of the genetic studies, a heterozygous missense mutation c.202 T>C, p.S68P (Ser68Pro) in exon 1 of the VHL gene that is mapped to chromosome 3p25.3, was found in the patients' DNA sample. The germline mutation of [c.202T $>$ C, p.S68P (Ser68Pro)] that was detected in both cases, has been reported in only two cases in the literature. However, in these reported cases, any systemic involvement except RH, were not reported. Although our cases had the same mutation, we detected renal involvement in both cases, and also central nervous system (CNS) involvement in one case, in addition to RH.

Keywords: Central nervous system (CNS) hemangioblastoma; Germline mutation; Renal cell

\footnotetext{
${ }^{1}$ Trakya University Faculty of Medicine, Department of Ophthalmology, Edirne, Turkey

${ }^{2}$ Trakya University Faculty of Medicine, Department of Medical Genetic, Edirne, Turkey
}

carcinoma; Retinal hemangioblastoma (RH); von Hippel-Lindau (VHL) disease.

\section{INTRODUCTION}

von Hippel-Lindau (VHL) (MIM\#193300) disease is an autosomal dominant inherited disorder that is classically associated with benign or malignant tumors in multiple organs. The most frequent tumors are retinal hemangioblastoma $(\mathrm{RH})$ and central nervous system (CNS) hemangioblastomas, renal cell carcinoma (RCC), pheochromocytoma, pancreatic islet cell tumors and endolymphatic sac tumours [1]. von Hippel-Lindau disease most commonly presents between the ages of 25 and 40, and the prevalence of the disease is 1 in 35000 [2]. Retinal hemangioblastoma is the most frequent neoplasm and the most common clinical sign of the disease; it is seen in 40.0$60.0 \%$ of patients [1]. Retinal hemangioblastomas are benign tumors that may be localized peripherally or juxtapapillary and show a slow-growing pattern [3].

In 1993, mutational inactivation of tumor suppressor genes in chromosome 3p25-p26 were found to be responsible for VHL [4]. The VHL coding sequence is represented in three exons and encodes two VHL proteins [5]. The $V H L$ gene functions as a tumor suppressor gene, and leads to neoplastic transformation when inactivated by the mutation that causes loss of function [6]. As a result of existing studies, many different $V H L$ gene point mutations, recombinations and deletions have been identified. In recent years, studies of patients with VHL have tried to establish 
the relationship between genotype and phenotype [7]. In this study, two cases of VHL from a single family are presented with renal and CNS involvement in addition to RH, which were not presented in this kind of mutation.

\section{CASE REPORT}

Case 1. A 29-year-old male patient was referred to the retina department. About 1 month previously, percutaneous radiofrequency ablation treatment was performed on the patient due to RCC in his left kidney (Figure 1). Central nervous system involvement was not detected in the patient.

On ophthalmological examination, best-corrected visual acuity (BCVA) was counting fingers from 2 meters in the right eye and 1.0 in the left eye on the Snellen acuity visual chart. Vitritis was detected in the right eye on slit-lamp biomicroscopy. The fundus examination revealed an extensive lipid exudation on the fovea and macula, and a 3-4 optic disc diametersized RH at the equator in the right eye; it was normal in the left eye. Fundus fluorescein angiography (FFA) showed that the RH in the right eye was responsible for fluorescein staining on the feeding artery and drainage vein and fluorescein leakage. Optical coherence tomography (OCT) showed that there was a foveal detachment in the right eye (Figure 2).

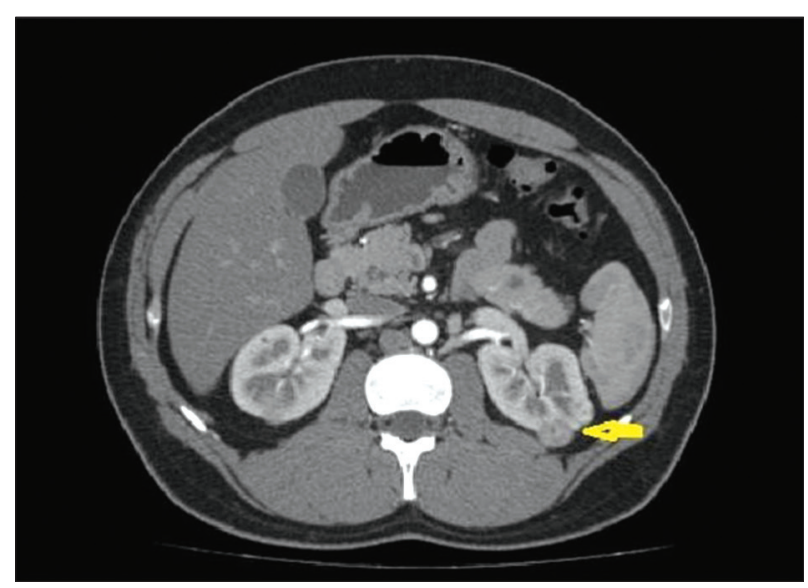

Figure 1. Abdominal computerized tomography image of case 1. Renal cell carcinoma in the left kidney (preoperatively).
The patient's blood sample was collected in vacutainers containing EDTA as anticoagulant. After DNA extraction (EZ1 Advanced Instruments; Qiagen, Hilden, Germany), mutation analysis of the $V H L$ gene (NG_008212.3, NM_000551) was performed using Sanger sequencing with the ABI PRISM ${ }^{\mathrm{TM}} 3130$ Avant system (Applied Biosystems, Grand Island, NY, USA). The entire coding region and the exon/intron boundaries of the VHL gene (transcript ENST00000256474) were sequenced. The amplicon panel primers and conditions used were established in the laboratory.

A heterozygous missense mutation c. $202 \mathrm{~T}>\mathrm{C}$, (p.Ser 68Pro) in exon 1 of the $V H L$ gene was found in the pa-tient's DNA sample (Figure 3). This mutation is reported in the HGMD-PUBLIC (CM073416) (http://www.hgmd. org).

The pathogenicity of the variation was tested in the Polyphen database and it was scored as probably damaging [probably damaging with a score of 0.960 (sensitivity: 0.78; specificity: 0.95)]. However, this variation was not listed the in the Exac database.

Case 2. A 33-year-old male patient (who is the first cousin of Case 1) was referred to the retina department. He was operated on because of a CNS hemangioblastoma 3 years earlier (Figure 4). The patient stated that he had vision loss in his left eye after the CNS operation. About 2 weeks earlier, a partial right nephrectomy operation was performed due to RCC (Figure 5).

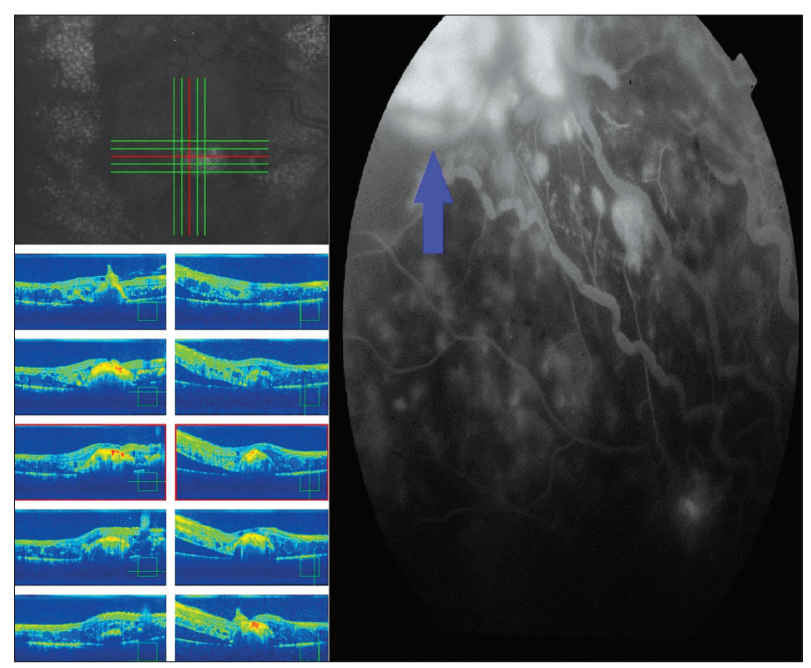

Figure 2. Optical coherence tomography and fundus fluorescein angiography images of Case 1.

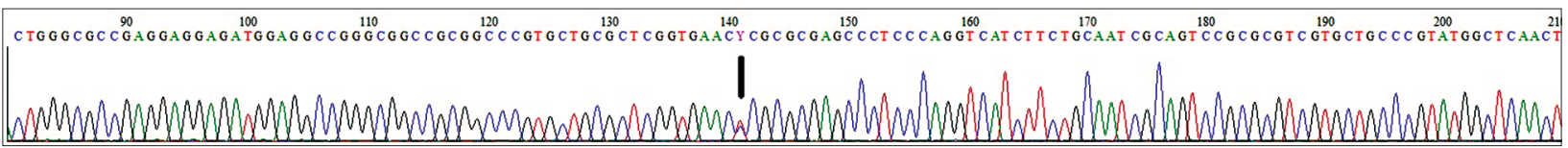

Figure 3. A heterozygous missense mutation c.202T $>$ C, (p.Ser68Pro) in exon 1 of the VHL gene. 


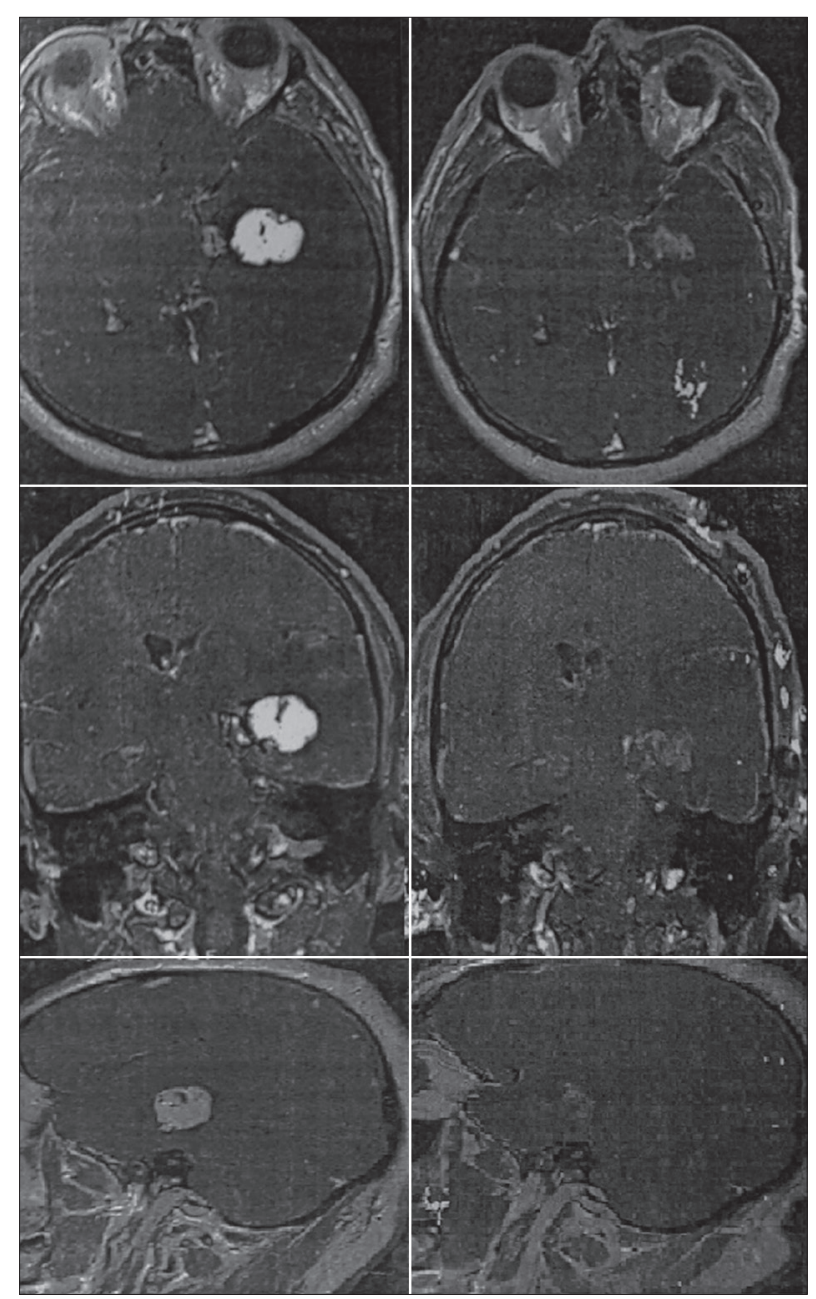

Figure 4. Preoperative and postoperative magnetic resonance images of the CNS haemangioblastoma (Case 2).
Best-corrected visual acuity was 1.0 on the Snellen acuity chart in the right eye and no light perception in the left eye. Biomicroscopic examination was normal in both eyes. The fundus examination revealed optic nerve atrophy and two $\mathrm{RH}$ at the equator in the left eye, and was normal in the right eye. Fundus fluorescein angiography showed that hyperfluorescence and fluorescein leakage was caused by the lesions. Hyper reflectance was observed under the cross-section of the RH, and the macular section was normal in OCT (Figure 6). The same mutation as in Case 1 was also detected in the genetic analysis (c.202T >C, p.Ser 68Pro).

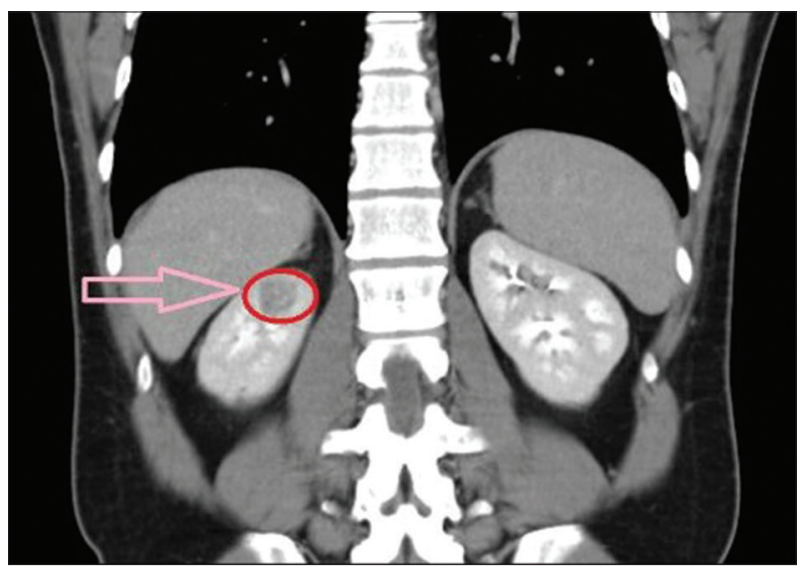

Figure 5. Abdominal computerized tomography image of Case 2. Renal cell carcinoma in the right kidney (preoperatively).

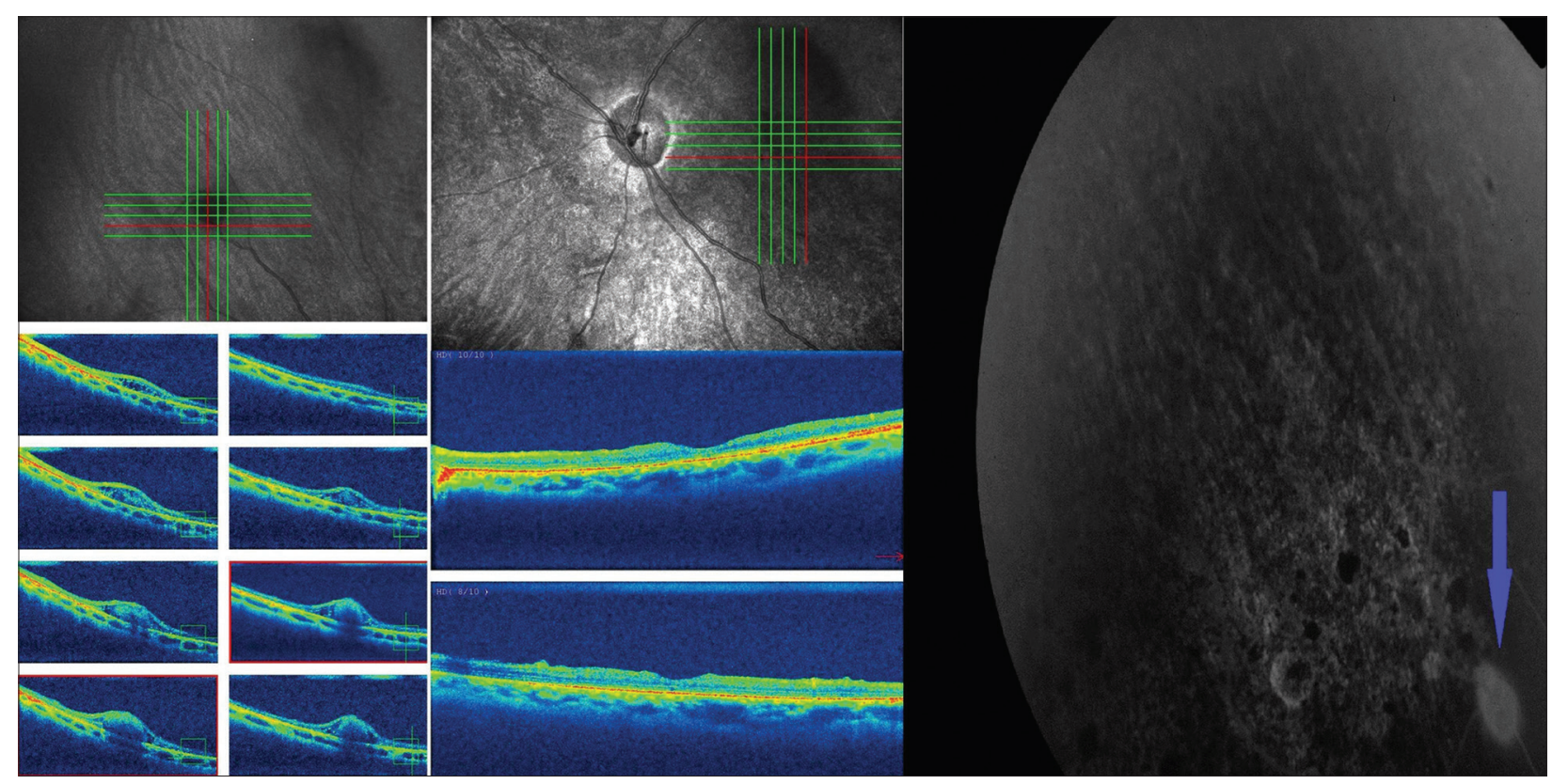

Figure 6. Optical coherence tomography and fundus fluorescein angiography images of Case 2. 


\section{DISCUSSION}

Fundus fluorescein angiography is the most informative diagnostic method because of the vascular nature of a RH. Fluorescein is apparent in the dilated feeding artery in the arterial phase, and the drainage vein becomes apparent in the venous phase, while the tumor demonstrates progressive hyperfluorescence with late leakage of dye into the surrounding structures [8]. In the two cases in this study, FFA examination showed that the typical feeding artery and drainage vein of the RH in the early-phase, and fluo-rescein leakage in the late-phase, was due to the vascular endothelial failure of the tumor.

The OCT findings in VHL have been published [9]. These include retinoschisis, epiretinal membranes, macular oedema and serous detachment. Optical coherence tomography demonstrated a foveal detachment in Case 1. The foveal detachment was due to fluid accumulation and macular oedema. Hyper reflectance was also observed under the cross-section of the RH upon OCT examination in Case 2. The authors consider that OCT is a useful diagnostic method in $\mathrm{RH}$ because it allows for the evaluation of peripheral tumors and macular complications, such as macular oedema, foveal detachment and epiretinal membranes.

Although RHs show a slow-growing pattern and are sometimes quiescent, they may lead to significant vision loss by causing cystoid macular oedema, and exudative and tractional retinal detachment [3]. It was believed that the primary reason for the vision loss in Case 1 was foveal detachment. The RH was close to the posterior pole in Case 1. In Case 2, the primary reason for the vision loss was optic nerve atrophy. The CNS hemangioblastoma operation may have led to optic nerve atrophy and vision loss in Case 2. Retinal hemangioblastomas were at the equator of the eye in Case 2. Germline mutations as a result of single nucleotide changes that were detected in these cases (c.202T $>$ C, p.Ser68Pro) are reported in only two other cases in the literature [10] but, in these cases, no systemic involvement except RH was detected. The other details of these cases have not been mentioned in the literature. Although these two cases had the same mutation, renal involvement was detected in both cases and also CNS involvement in Case 2, in addition to RH.

Life-threatening diseases such as VHL can be diagnosed by ophthalmologists. The life expectancy of VHL patients is below the age of 50 because of RCC and CNS hemangioblastoma complications [11]. von Hippel-Lindau disease is a familial cancer syndrome; patients with VHL and at-risk family members of these patients should be followed by periodic screening programs and genetic analyses. Eventually, the development of early diagnosis methods in VHL, such as genetic analysis, may provide both superior visual prognosis and life expectancy. In conclusion, VHL patients who had a germline mutation resulting from a single nucleotide change in the VHL gene (c.202 T>C, p.Ser68Pro) missense mutation may have renal and CNS involvement, in addition to $\mathrm{RH}$.

\section{ACKNOWLEDGMENTS}

This study was presented at the 48th National Congress of Turkish Ophthalmology Society, held at Antalya, Turkey on 5-9 November 2014. [Poster Presentations (PS-1103)].

Declaration of Interest. The authors report no conflicts of interest. The authors alone are responsible for the content and writing of this article.

\section{REFERENCES}

1. Maher ER, Yates JR, Harries R, Benjamin C, Harris R, Moore AT, et al. Clinical features and natural history of von Hippel-Lindau disease. Q J Med. 1990; 77(283): 1151-1163.

2. Singh AD, Shields CL, Shields JA. von HippelLindau disease. Surv Ophtalmol. 2001; 46(2): 117-142.

3. Kreusel KM, Bechrakis NE, Krause L, Neumann HP, Foerster MH. Retinal angiomatosis in von Hippel-Lindau disease: A longitudinal ophthalmologic study. Ophthalmology. 2006; 113(8): 1418-1424.

4. LatifF, Tory K, Gnarra J, Yao M, Duh FM, Orcutt ML, et al. Identification of the von HippelLindau disease tumor suppressor gene. Science. 1993; 260(5112): 1317-1320.

5. Woodward ER, Buchberger A, Clifford SC, Hurst LD, Affara NA, Maher ER. Comparative sequence analysis of the VHL suppressor gene. Genomics. 2000; 65(3): 253-265. 
6. Harbour JW. Tumor suppressor genes in ophthalmology. Surv Ophthalmol. 1999; 44(3): 235-246.

7. Wong WT, Agron E, Coleman HR, Reed GF, Csaky K, Peterson J, et al. Genotype-phenotype correlation in von Hippel-Lindau disease with retinal angiomatosis. Arch Ophthalmol. 2007; 125(2): 239-245.

8. Welch RB. Fluorescein angiography in sicklecell retinopathy and von Hippel-Lindau disease. Int Ophthalmol Clin. 1977; 17(2): 137-154.

9. Sato A, Fukui E, Ohta K. Retinoschisis detected by spectral-domain optical coherence tomography in von Hippel-Lindau disease. Retin Cases Brief Rep. 2012; 6(3): 317-319.
10. Hes FJ, van der Luijt RB, Janssen AL, Zewald RA, de Jong GJ, Lenders JW, et al. Frequency of Von Hippel-Lindau germline mutations in classic and non-classic Von Hippel-Lindau disease identified by DNA sequencing, Southern blot analysis and multiplex ligation-dependent probe amplification. Clin Genet. 2007; 72(2): 122-129.

11. Chew EY. Ocular manifestations of von HippelLindau disease: Clinical and genetic investigations. Trans Am Ophthalmol Soc. 2005; 103: 495-511. 
